OPEN ACCESS

Edited by:

Giulia Roda,

Humanitas University, Italy

Reviewed by:

Zubair Khan,

University of Texas Health Science

Center at Houston, United States

Vik Meadows,

Indiana University, United States

*Correspondence:

David Dunkin

david.dunkin@mssm.edu

tThese authors have contributed equally to this work

Specialty section:

This article was submitted to

Gastroenterology,

a section of the journal

Frontiers in Medicine

Received: 24 September 2021 Accepted: 02 November 2021 Published: 29 November 2021

Citation:

Chen $X$, Lai J, Song $Y$, Yang $N$, Gnjatic S, Gillespie V, Hahn W,

Chefitz E, Pittman N, Jossen J,

Benkov K, Dubinsky M, Li X-M and Dunkin D (2021) Butanol Purified Food

Allergy Herbal Formula-2 Has an Immunomodulating Effect ex-vivo in Pediatric Crohn's Disease Subjects.

Front. Med. 8:782859.

doi: 10.3389/fmed.2021.782859

\title{
Butanol Purified Food Allergy Herbal Formula-2 Has an Immunomodulating Effect ex-vivo in Pediatric Crohn's Disease Subjects
}

\section{Xin Chen ${ }^{1,2 \dagger}$, Joanne Lai $^{1 \dagger}$, Ying Song ${ }^{3}$, Nan Yang ${ }^{4,5,6}$, Sacha Gnjatic ${ }^{6,7}$, Virginia Gillespie ${ }^{8}$, William Hahn ${ }^{1}$, Ezra Chefitz ${ }^{1}$, Nanci Pittman ${ }^{1}$, Jacqueline Jossen ${ }^{1}$, Keith Benkov ${ }^{1}$, Marla Dubinsky ${ }^{1}$, Xiu-Min Li ${ }^{5,6}$ and David Dunkin ${ }^{1,2 *}$}

\footnotetext{
${ }^{1}$ Division of Pediatric Gastroenterology, The Icahn School of Medicine at Mount Sinai (ISMMS), New York, NY, United States, ${ }^{2}$ The Mindich Child Health and Development Institute (MCHDI), The Icahn School of Medicine at Mount Sinai (ISMMS), New York, NY, United States, ${ }^{3}$ Academy of Chinese Medicine, Henan University of Chinese Medicine, Zhengzhou, China, ${ }^{4}$ Microbiology and Immunology, New York Medical College, Valhalla, NY, United States, ${ }^{5}$ General Nutraceutical Technology, Elmsford, NY, United States, ${ }^{6}$ Division of Hematology and Medical Oncology, New York, NY, United States, ${ }^{7}$ The Precision Immunology Institute, The Icahn School of Medicine at Mount Sinai (ISMMS), New York, NY, United States, ${ }^{8}$ Department of Comparative Pathology, The Icahn School of Medicine at Mount Sinai (ISMMS), New York, NY, United States
}

Background: TNF- $\alpha$ has a major role in the pathogenesis of Crohn's disease (CD). In contrast, GM-CSF may be beneficial for its anti-inflammatory role in a subset of patients with CD with antibodies against GM-CSF as seen in prior trials of GM-CSF which resulted in clinical improvement in $\mathrm{CD}$. We developed butanol purified Food Allergy Herbal Formula-2 (B-FAHF-2) by refining FAHF-2. FAHF-2 suppressed TNF- $\alpha$ production by human peripheral blood mononuclear cells (PBMCs) and colonic mucosa, and abrogated colitis in a murine model. We sought to examine the effect of B-FAHF-2 and the herbs that comprise it on TNF- $\alpha$ and GM-CSF production as a potential herbal therapy for the treatment of $\mathrm{CD}$.

Methods: B-FAHF-2 was examined using high pressure liquid chromatography (HPLC) and compared to the original formulation, FAHF-2. PBMCs from pediatric patients with CD were cultured with lipopolysaccharide and B-FAHF-2, individual herbs or medium alone. Colonic biopsy specimens were cultured with or without B-FAHF-2. TNF- $\alpha$ and GM-CSF were measured by enzyme-linked immunosorbent assay (ELISA). B-FAHF-2 efficacy was tested in vivo in the CD45Rb ${ }^{\text {hi }}$ transfer model.

Results: B-FAHF-2 had a similar HPLC fingerprint as FAHF-2 but decreased TNF- $\alpha$ production by PBMCs and colonic mucosa from pediatric CD subjects at $20 \%$ of the FAHF-2 dose. B-FAHF-2 increased GM-CSF production by PBMCs and colonic mucosa from pediatric CD subjects including those with antibodies to GM-CSF. Of B-FAHF-2's herbal constituents, only Huang Bai suppressed TNF- $\alpha$ and increased GM-CSF production. In the murine model, B-FAHF-2 treatment alleviated colitis. 


\begin{abstract}
Conclusions: B-FAHF-2 decreased TNF- $\alpha$ production by PBMCs and colonic mucosa from pediatric subjects at a lower dose than FAHF-2. B-FAHF-2 also increased GM-CSF production by PBMCs independent of antibodies. B-FAHF-2 may have a benefit in CD patients.
\end{abstract}

Keywords: Crohn's disease, herbal therapy, pre-clinical drug testing, immunomodulatory, drug development

\section{INTRODUCTION}

Crohn's disease (CD), a form of inflammatory bowel disease (IBD), is a life-long disease characterized by chronic and relapsing inflammation of the gastrointestinal tract. The prevalence of $\mathrm{CD}$ varies based on region, race, age, and environment. The annual incidence of CD ranges from 16.7 to 318.5 per 100,000 in North American, 0.6 to 322 per 100,000 in Europe, and 0.88 to 67.9 per 100,000 in Asia and the Middle East (1). Among CD patients, 20-25\% are diagnosed before 17 years old. The incidence of pediatric CD is steadily increasing (2). CD has multiple clinical phenotypes and disease severities that determine which therapy is utilized. Currently, there are numerous treatment options for children and adults with moderate-to-severe $\mathrm{CD}$, but few that are approved to treat those with mild-to-moderate disease. The only FDA approved medication for treatment of mild-to-moderate CD in children 8 years and older is Entocort EC (budesonide). The indications allow for up to 10 weeks of use in active disease but not for use as a maintenance therapy in children. Budesonide is a steroid compound and as such may have the same side effects as corticosteroids. Other medications including immunomodulators and biologics are used off-label for mildto-moderate $\mathrm{CD}$ in children and have risks for significant adverse effects. Thus, there is a need for the development of new therapies.

Herbal therapies may fill this therapeutic void and there is a growing interest in complementary treatments (3). Food Allergy Herbal Formula-2 (FAHF-2) is composed of nine Chinese herbal medications that are originally part of the traditional Chinese herbal formula, Wu Mei Wan, that has been used safely to treat colitis in China and Japan for thousands of years (4). FAHF-2 has been shown in both murine and human studies of food allergy to inhibit both adaptive and innate immune pro-inflammatory cytokine responses in peripheral blood mononuclear cells (PBMCs) and in vivo (5-10). Interestingly, in food allergic subjects, FAHF-2 induced potent suppression of TNF- $\alpha$, one of the major inflammatory cytokines involved in the pathogenesis of IBD. Anti-TNF antibody-based biologics are one of the most efficacious groups of medications currently in use to treat IBD. That led to our investigation on the effects of FAHF-2 in IBD. We showed that FAHF- 2 suppresses TNF- $\alpha$, IFN- $\gamma$, IL- $1 \beta$, IL6 , IL-12 and IL-17 production from both PBMCs and inflamed colonic mucosa from pediatric subjects with $\mathrm{CD}$, and abrogates

Abbreviations: FAHF-2, Food Allergy Herbal Formula-2; B-FAHF-2, butanol purified FAHF-2; IBD, inflammatory bowel disease; CD, Crohn's disease; HPLC, high pressure liquid chromatography; PBMCs, Peripheral blood mononuclear cells. murine colitis in the CD45RB ${ }^{\text {hi }}$ transfer model (5). FAHF-2 affects immune responses by disrupting the NF-kB pathway (5).

FAHF-2's effects are immunomodulatory and not globally immunosuppressive as evidenced by the effect FAHF-2 has on the production of IFN- $\gamma$ in different inflammatory diseases. In food allergy, FAHF-2 stimulates increased production of $\operatorname{IFN}-\gamma(8,11)$, whereas in $\mathrm{CD}$ it decreases IFN- $\gamma$ production (5). In addition, FAHF-2's effects on chemokines and growth factors shown here for the first time, demonstrate that FAHF-2 suppresses production of numerous chemokines (IP-10, MIP-1 $\beta$, eotaxin, RANTES, MIG, MCP-1, and MIP-1 $\alpha$ ) and stimulates production of granulocyte-macrophage colony-stimulating factor (GM-CSF) by PBMCs from pediatric subjects with CD.

GM-CSF in particular plays an integral role in intestinal innate immunity, immune tolerance and the induction of intestinal regulatory $\mathrm{T}$ cells $(12,13)$. Pediatric and adult $\mathrm{CD}$ patients with ileal and stricturing or penetrating disease were shown to have higher levels of GM-CSF autoantibodies (14). A recombinant human form of GM-CSF, sargramostim, had positive effects in a small pilot study of CD patients. A subsequent larger placebo-controlled trial showed that GM-CSF therapy decreased disease severity and induced mucosal healing, but failed to demonstrate a clinical response (15). The larger study failed to target subjects with deficiencies or antibodies against GM-CSF and this may have been the reason for its failure. Heterogeneity in pathogenic mechanisms exists. Thus, an increase in GMCSF may be therapeutic for a subgroup of CD patients, and we therefore continued to investigate the ability of FAHF-2 and its derivatives to alter GM-CSF.

FAHF-2 shows promise as a possible therapy for CD. Clinical trials of FAHF-2 in food allergy however, revealed that the high burden of pills needed in clinical trials contributed to non-compliance (16). Our goal was to lower the pill burden needed to use FAHF-2 in treatment protocols. We therefore developed butanol purified FAHF-2 (B-FAHF-2) which could be used at a fraction of the dose. We also tested the individual herbs that comprise FAHF-2 and B-FAHF-2 for their effects on TNF- $\alpha$ and GM-CSF as representative of their ability to modulate the immune response in $\mathrm{CD}$. We found that the purified form, B-FAHF-2, significantly suppressed the production of TNF- $\alpha$ by PBMCs and intestinal mucosal from pediatric subjects with $\mathrm{CD}$ and abrogated colitis in a murine model at a much lower dose than FAHF-2. BFAHF-2 also increased production of GM-CSF by PBMCs and intestinal mucosa and this was also true in a small subset of our CD subjects with antibodies against GMCSF. Consistent with traditional Chinese medicine practices, the individual herbs within FAHF-2 and B-FAHF-2 were 
TABLE 1 | The composition of FAHF-2.

\begin{tabular}{lllc}
\hline Latin name & Chinese name & Plant part & $\begin{array}{c}\text { Occupied } \\
\text { percent }\end{array}$ \\
\hline Prunus mume & Wu-Mei & Fruit & $20 \%$ \\
Zanthoxylum schinifolium & Chuan-Jiao & Pericarp & $2 \%$ \\
Angelica sinensis & Dang-Gui & Root & $6 \%$ \\
Zingiber officinalis & Gan-Jiang & Root & $6 \%$ \\
Cinnamomum cassiae & Gui-Zhi & Burgeon & $4 \%$ \\
Phellodendron chinense & Huang-Bai & Cortex & $4 \%$ \\
Coptis chinensis & Huang-Lian & Root & $6 \%$ \\
Panax ginseng & Hong-Shen & Root & $6 \%$ \\
Ganoderma lucidum & Ling-Zhi & Mushroom & $46 \%$ \\
\hline
\end{tabular}

not as effective as the whole in suppressing TNF- $\alpha$ and increasing GM-CSF.

\section{MATERIALS AND METHODS}

\section{FAHF-2 and B-FAHF-2 Production, Quality Control, and Dose Derivation}

FAHF-2 and B-FAHF-2 were obtained from Xiyuan Chinese Medicine Research and Pharmaceutical Manufacturer, Chinese Academy of Chinese Medicine Sciences, Beijing, China, a good manufacturing practice (GMP) certified facility. Herbal components of FAHF-2 are listed in Table 1. All herbs were inspected for identity and quality by licensed pharmacists. All plant names have been checked with http://www.theplantlist.org except for Ganoderma lucidum which is a fungus and not listed there. Voucher specimens of the raw herbs were archived in the laboratory of Dr. Xiu-Min Li. The manufacturing process of FAHF2 is as follows: Ganoderma lucidum powder was generated by decoctions by boiling small pieces Ganoderma lucidum twice ( $2 \mathrm{~h}$ each time). Panax ginseng was extracted twice with $80 \%$ aqueous ethanol, then filtered, combined, and evaporated until there was no residual ethanol. The other 7 herbs were combined and boiled twice in water ( $1.5 \mathrm{~h}$ each time). The decoction was collected, combined, and purified by ethanol precipitation. Panax ginseng was mixed with the 7 herbs extract, dried into a powder, and then combined with the Ganoderma lucidum powder to form FAHF-2 $(17,18)$.

Extensive quality control, analytical chemistry data and batch consistency pertaining to the formula have been published previously (11). We identified chemical markers by high-pressure liquid chromatography (HPLC) and liquid chromatography (LC) mass spectrometry. Endotoxin levels were measured using the Pyrogent Plus assay kit (Lonza, MA) and were below $0.03 \mathrm{EU} / \mathrm{ml}$, the limit of sensitivity for this kit.

Given the generally favorable safety profile, the dose ranges of most Chinese herbal formulations are wide. Initially, the dose used in our FAHF-2 studies was converted from the human daily dose of the Wu Mei Wan formula and Ling Zhi extract at a medium to high dose range using a conversion table of equivalent effective dose ratios from humans to animals based on body surface area (19). This dose was consistently effective in protecting peanut allergic mice from anaphylaxis, and at abrogating colitis in a murine model (5). B-FAHF-2 is $20 \%$ of FAHF-2 by weight because of the elimination of non-medicinal components and thus the dose used in our experiments is derived by using $20 \%$ of the dose of FAHF-2 $(250 \mu \mathrm{g} / \mathrm{ml})$. The effective dose $(60 \mu \mathrm{g} / \mathrm{ml})$ of B-FAHF-2 was found to be $20 \%$ that of FAHF2 based upon cell culture assays and in vivo models of peanut allergy (6). For the individual herbs within FAHF-2 and B-FAHF2 , the doses used correspond to the equivalent dose found in the full compound. Equivalent doses were as follows: Wu Mei (WM) $50 \mu \mathrm{g} / \mathrm{ml}$, Chuan Jiao (HJ) $125 \mu \mathrm{g} / \mathrm{ml}$, Dang Gui (DJ) $125 \mu \mathrm{g} / \mathrm{ml}$, Gan Jiang (GJ) $125 \mu \mathrm{g} / \mathrm{ml}$, Gui Zhi (GZ) $125 \mu \mathrm{g} / \mathrm{ml}$, Huang Bai (HB) $125 \mu \mathrm{g} / \mathrm{ml}$, Huang Lian (HL) $125 \mu \mathrm{g} / \mathrm{ml}$, Hong Shen (HS) $125 \mu \mathrm{g} / \mathrm{ml}$, and Ling Zhi (LZ) $125 \mu \mathrm{g} / \mathrm{ml}$.

\section{HPLC of FAHF-2, and B-FAHF-2}

The instruments used were a Waters 2690 HPLC system coupled to a 2996 PDA detector (Waters, Milford, MA). B-FAHF-2 tablets were ground, and a $22.5 \mathrm{mg} / \mathrm{mL}$ solution was prepared using 1:1 ratio of mobile phase mixture. The B-FAHF-2 solution was centrifuged at $10,000 \mathrm{rpm}$ for 10 minutes. $10 \mu \mathrm{L}$ of the supernatant was injected into the HPLC system and separated on a ZORBAX SB-C18 $(4.6 \times 150 \mathrm{~mm}, 5 \mu \mathrm{m})$ column (Agilent, Santa Clara, CA). The mobile phase A was made of $0.1 \%$ of formic acid aqueous solution. Mobile phase B was acetonitrile. The separation was performed at $1 \mathrm{~min} / \mathrm{mL}$ flow rate following a linear gradient elution of $2-25 \%$ mobile phase B in $45 \mathrm{~min}$, $25-35 \% \mathrm{~B}$ in the following $25 \mathrm{~min}, 35-55 \%$ in the next $15 \mathrm{~min}$, $55-75 \%$ in another $10 \mathrm{~min}$. This mobile phase composition was maintained for $5 \mathrm{~min}$ and then rapidly switched to $2 \%$ mobile phase B. An equivalent amount of FAHF-2 formula, 99 $\mathrm{mg} / \mathrm{mL}$, was also prepared following the same procedure as for B-FAHF-2. $10 \mu \mathrm{L}$ of FAHF-2 supernatant was analyzed on the HPLC system. Data was collected and processed with Waters' Empower software.

\section{Subjects}

Human studies were approved by the Institutional Review Board of the Icahn School of Medicine at Mount Sinai (No. 11-00808). All participants provided written informed consent for participation in the study. Blood samples $(n=$ 29 ) and inflamed colonic biopsy specimens $(n=20)$ were collected from newly diagnosed pediatric CD patients (819 years old) naïve to medications that could alter immune responses including: immune-modulators, biologics or steroids. CD patients were diagnosed based on standard clinical, radiographic and endoscopic criteria. Blood and colonic biopsy specimens were also collected from non-IBD control subjects ( $n$ $=12$ ). Patient characteristics are outlined in Table 2 .

\section{PBMC Separation, Cell Culture and Cytokine, Chemokine, and Growth Factor Measurements}

PBMCs were isolated by Ficoll Hypaque (Pharmacia, Piscataway, NJ) with density gradient centrifugation. Purified PBMCs were cultured in medium containing 5\% FBS with or without FAHF-2 $(250 \mu \mathrm{g} / \mathrm{ml})$, B-FAHF-2 $(60 \mu \mathrm{g} / \mathrm{ml})$, individual herbs 
TABLE 2 | Subject characteristics based upon the Montreal classification for CD.

\begin{tabular}{lll}
\hline & CD $(\boldsymbol{n}=\mathbf{2 9})$ & Non-IBD $(\boldsymbol{n}=\mathbf{1 2})$ \\
\hline Gender (male/female) & $21 / 8$ & $6 / 6$ \\
Age, mean $\pm \mathrm{SD}$ & $13 \pm 3.2$ & $15.8 \pm 2.5$ \\
Location (L2/L3) & $6 / 23$ & $\mathrm{~N} / \mathrm{A}$ \\
Behavior (B1/B2/B3) & $26 / 0 / 3$ & $\mathrm{~N} / \mathrm{A}$ \\
Perianal (N/Y) & $26 / 3$ & $\mathrm{~N} / \mathrm{A}$ \\
\hline
\end{tabular}

L2, colonic; $L 3$, ileocolonic; $B 1$, non-stricturing, non-penetrating; $B 2$, stricturing; $B 3$, penetrating.

TABLE 3 | Real-time PCR primer sequences.

\begin{tabular}{|c|c|c|}
\hline Target & Forward sequences $\left(5^{\prime}-3^{\prime}\right)$ & Reverse sequences $\left(5^{\prime}-3^{\prime}\right)$ \\
\hline TGF- $\beta 1$ & CCCGAAGCGGACTACTATGC & CGAATGTCTGACGTATTGAAGAAC \\
\hline TGF- $\beta 2$ & CACCCAGCGCTACATCGATAG & CAGCGTCTGTCACGTCGAA \\
\hline |L-10 & ПTGAATTCCCTGGGTGAGAA & GCTCCACTGCCTTGCTCTTATT \\
\hline Foxp3 & ACTGGGGTCTTCTCCСTCAA & CGTGGGAAGGTGCAGAGTAG \\
\hline Gapdh & GGTGGTCTCCTCTGACTTCAAC & GTTGCTGTAGCCAAATTCGTTGT \\
\hline
\end{tabular}

in B-FAHF-2 (doses noted above) or dexamethasone $\left(10^{-3}\right.$ $\mu \mathrm{M} / \mathrm{ml}$, positive control) for $24 \mathrm{~h}$ followed by LPS $(2 \mu \mathrm{g} / \mathrm{ml})$ stimulation or no stimulation for an additional $24 \mathrm{~h}$ (8). Cytokine, chemokine and growth factor levels in the culture supernatants were determined by ELISA per the manufacturer's instructions (BD Biosciences), by multiplex immunoassay or by cytometric bead array. Multiplex assays were performed per the manufacturer's instructions (Luminex Human assay, Invitrogen, Grand Island, NY). Cytometric bead array was performed per the manufacturer's instructions (BD Biosciences).

\section{Biopsy Preparation and Culture}

Colonic biopsies were washed with PBS and then cultured with or without B-FAHF-2 $(60 \mu \mathrm{g} / \mathrm{ml})$ in complete RPMI (10\% FBS and GPS) with phosphatase and protease inhibitors overnight. The volume of culture medium was based upon the weight of each sample so that the size of the biopsies could be standardized. Supernatants were filtered and TNF- $\alpha$ and GM-CSF were assessed by ELISA (BD Biosciences) per the manufacturer's instructions.

\section{GM-CSF Antibody Measurement}

Serum was tested by ELISA. Briefly, samples were diluted serially in 4-fold increments from $1 / 100$ to $1 / 25,000$ and assayed for the presence of IgG and IgA against GM-CSF (Sargramostim, Genzyme), as well as irrelevant control recombinant proteins. Each plate included positive control sera from patients with pulmonary alveolar proteinosis as well as a negative control healthy donor serum pool, expected, respectively, to react or not with GM-CSF.

\section{CD45RB $^{\text {hi }}$ T Cell Transfer Model of Colitis}

Animal studies were approved by the Institutional Animal Care and Use Committee at Mount Sinai. Cells were obtained from C57BL/6 wild type mice and enriched using an EasySep Cell Isolation Kit (STEMCELL Technologies Inc., Vancouver, CA) that depletes $\mathrm{CD}^{+}, \mathrm{CD} 11 \mathrm{~b}^{+}, \mathrm{CD} 11 \mathrm{c}^{+}, \mathrm{CD} 19^{+}$, and $\mathrm{B} 220^{+}$cells by negative selection. The resulting $\mathrm{CD}^{+}$enriched population was labeled with FITC-conjugated anti-CD4 Ab, APC-conjugated anti-CD62L $\mathrm{Ab}$ and $\mathrm{PE}$-conjugated anti$\mathrm{CD} 45 \mathrm{RB} \mathrm{Ab}$ (eBiosciences). Subpopulations of $\mathrm{CD} 4^{+}$cells were sorted by flow cytometry. CD4 ${ }^{+}$CD 45 RBhi T cells $\left(3.5 \times 10^{5}\right)$ were adoptively transferred by intraperitoneal injection into recipient $\mathrm{RAG}^{-/-}$mice. Transfer was confirmed by flow cytometry of peripheral blood in all mice after 2-4 weeks. To prevent the progression to colitis, the recipient mice were fed BFAHF-2 (50 mg/day) daily by gavage $24 \mathrm{~h}$ after CD $4{ }^{+} \mathrm{CD} 45 \mathrm{RBhi}$ $\mathrm{T}$ cells transfer. Control mice received water by gavage. Weights were recorded semi-weekly. All mice were sacrificed once any mouse lost $20 \%$ of their initial weight. Colonic histology was scored for inflammatory infiltrates and epithelial damage by a pathologist blinded to the treatment group $(20,21)$. A total score with a maximum of 20 was determined by the summation of the following sub-scores: mucosal involvement: $0=$ normal, 1 $=3-10$ mucosal neutrophils $/ \mathrm{hpf}, 2=$ more than 10 mucosal neutrophils or rare crypt abscesses, $3=$ multiple crypt abscesses or erosion; submucosal involvement: $0=$ normal, $1=$ focal aggregates of neutrophils, $2=$ neutrophil infiltration with expansion of submucosa, 3 = diffuse neutrophil infiltration; muscularis: $0=$ normal, $1=$ scattered neutrophils within the muscularis, 2 = neutrophil infiltration with focal effacement of the muscularis, $3=$ extensive neutrophil infiltration with transmural effacement of the muscularis; crypt damage: $0=$ normal, $1=$ loss of the basal one third, $2=$ loss of the basal two thirds, $3=$ entire crypt loss, $4=$ crypt loss with surface erosion, $5=$ confluent, extensive erosion; ulcerations: $0=$ absence of ulceration, $1=$ one to two ulcer foci, $2=$ three to four ulcer foci, 3 = confluent extensive ulceration. Colonic samples were cultured overnight in medium containing phosphatase and protease inhibitors. Cytokine secretion was measured in the supernatants (IL-4, IL-6, IL-10, IL-12, TNF- $\beta$, IFN- $\gamma$, IL17A) by cytometric bead array (BD Biosciences) and ELISA for GM-CSF (BD Biosciences) per the manufacturer's instructions. RT-PCR was performed to look at regulatory elements within the colonic mucosa including TGF $\beta 1$, TGF 32 , Foxp3, and IL-10. Colon samples' RNA extraction and complementary DNA (cDNA) transcription were performed as previously described. (22) SYBR ${ }^{\mathrm{TM}}$ Green Master Mix (Thermo Fisher Scientific, Fair lawn, NJ) was used to perform RT-PCR. The target gene mRNA expression was normalized to the untreated group and calculated with the ${ }_{\Delta \Delta}$ CT method. The primer sequences are listed in Table 3. Immunofluorescence (IF) staining of mouse colon sections was performed according to the previously described protocol with slight modifications (23). Paraffin embedded slides were dewaxed by serial xylene, xylene/ethanol (1:1), 100\% ethanol, 95\% ethanol, 70\% ethanol, and $\mathrm{H}_{2} \mathrm{O}$. Then slides were unmasked in heat antigen retrieval solution in the microwave. After permeabilization for $10 \mathrm{~min}$ at room temperature (RT), the slides were blocked with $20 \%$ goat serum in PBS for $1 \mathrm{~h}$ at room temperature, and then incubated with CD4-FITC and F4/80-APC (eBiosciences, 


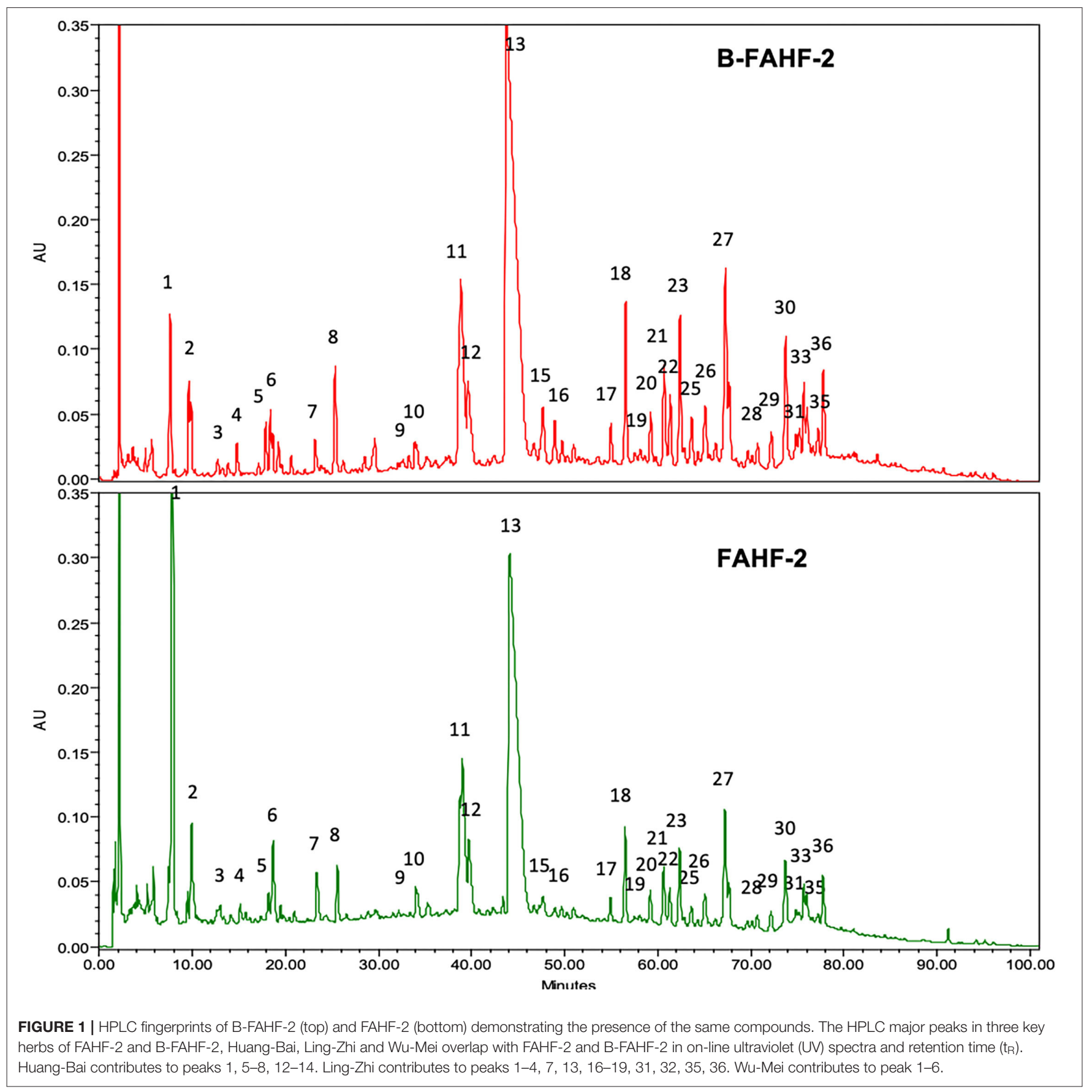

San Diego, CA) overnight at $4^{\circ} \mathrm{C}$. DAPI was used to stain cell DNA.

\section{Statistics}

Paired T-tests with Wilcoxon signed-rank test were used when comparing the same samples without and with treatment. Comparisons between multiple groups were done using one-way ANOVA. This was followed by either non-parametric
Mann-Whitney U test or Bonferroni analysis when appropriate. Statistical differences between groups for the colitis model were determined by Mann-Whitney T-test assuming nonnormally distributed data. Data analysis was done using Prism software (GraphPad, San Diego, CA). A value of $p$ $<0.05$ was considered statistically significant. $P$-values are indicated by ${ }^{*} p<0.05,{ }^{* *} p<0.01,{ }^{* * *} p<0.001,{ }^{* * * *} p$ $<0.0001$. 


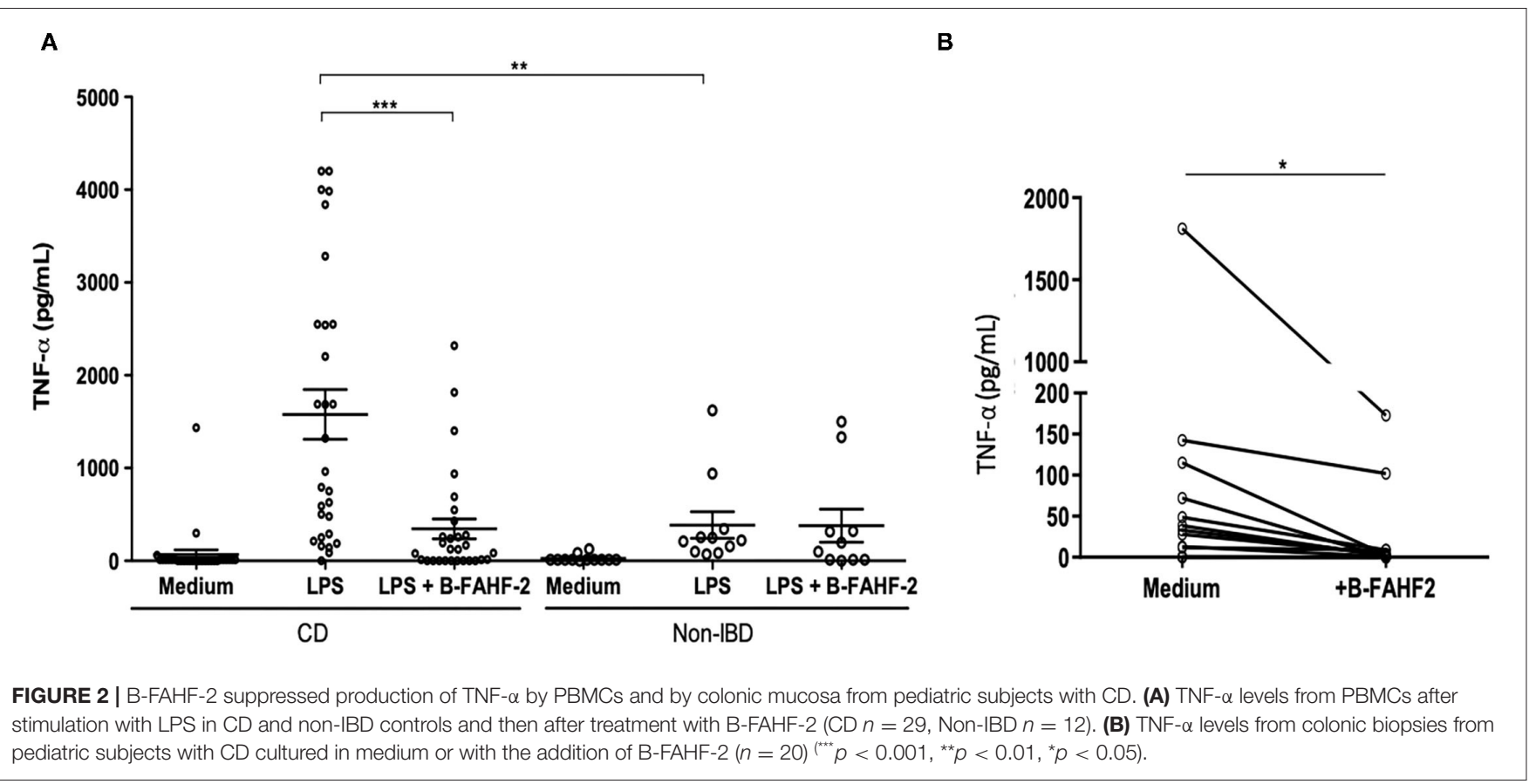

\section{RESULTS}

\section{B-FAHF-2 Contained the Same Compounds as FAHF-2 and Suppressed TNF- $\alpha$ Production by PBMCs and Colonic Mucosa From Pediatric Subjects With CD at $\mathbf{2 0} \%$ of the Dose of FAHF-2}

After butanol extraction of FAHF-2 was completed, B-FAHF2 and FAHF-2 were examined by HPLC to ensure that the compounds were similar. HPLC fingerprints (Figure 1) demonstrate similar patterns with peaks at similar absorbance units and retention times, indicating that they contain the same compounds.

FAHF-2 suppressed production of TNF- $\alpha$ IFN- $\gamma$, and IL-12 from PBMCs from pediatric subjects with $\mathrm{CD}$ (5) and therefore we examined B-FAHF-2's effects on TNF- $\alpha$ production as a representative cytokine. We first tested the effect of B-FAHF-2 $(60 \mu \mathrm{g} / \mathrm{ml})$ on TNF- $\alpha$ secretion by PBMCs from 29 CD subjects and 12 non-IBD controls (Table 2). PBMCs from CD subjects secreted significantly more TNF- $\alpha$ upon LPS stimulation as compared to non-IBD controls $(p<0.01)$ (Figure 2A). TNF- $\alpha$ levels were significantly reduced when CD PBMCs were cultured with B-FAHF-2 $(p<0.001)$ (Figure 2A). No cytotoxicity was detected (Data not shown).

To be effective at treating CD, B-FAHF-2 should have effects on intestinal mucosa and inflammatory cytokine secretion. FAHF-2 suppressed production of TNF- $\alpha$, IL-1 $\beta$, IL-6, and IL17 from inflamed colonic mucosa from pediatric subjects with CD (5) and therefore we examined B-FAHF-2's effects on TNF- $\alpha$ production as a representative cytokine. We incubated inflamed colonic mucosa from subjects with $\mathrm{CD}$ with and without $\mathrm{B}$ FAHF- 2 and quantified TNF- $\alpha$ from the supernatant. B-FAHF-2 significantly suppressed the production of TNF- $\alpha$ from inflamed biopsies from subjects with CD $(p<0.05)$ (Figure 2B).

Thus, like FAHF-2, B-FAHF-2 significantly suppressed TNF$\alpha$ production by PBMCs and inflamed colonic mucosa from pediatric subjects with $C D$.

\section{B-FAHF-2 Stimulated Increased Production of GM-CSF by PBMCs and Colonic Mucosa From Pediatric Subjects With CD}

FAHF-2 had an immunomodulatory effect that is best demonstrated by its stimulation of increased production of GM-CSF while at the same time suppressing production of multiple inflammatory chemokines (IP-10, MIP-1 $\beta$, eotaxin, RANTES, MIG, MCP-1, and MIP-1 $\alpha$ ) and HGF from PBMCs from pediatric subjects with CD (Figure 3).

Therefore, using GM-CSF as representative of the immunomodulatory capacity FAHF-2, we tested the effect of B-FAHF-2 on GM-CSF secretion by unstimulated PBMCs from all 29 pediatric subjects with CD. PBMCs cultured with B-FAHF-2 produced more GM-CSF than when cultured in medium alone (Figure 4A) $(p<0.05)$. B-FAHF-2 also stimulated significantly increased production of GM-CSF from biopsies from pediatric subjects with CD (Figure 4B) $(p<0.05)$.

\section{B-FAHF-2 Increased GM-CSF Production by PBMCs From Pediatric Subjects With CD Who Have GM-CSF Antibodies}

Given the effect of B-FAHF-2 on GM-CSF and the possibility that it might be beneficial in a subset of patients with GM-CSF antibodies, we tested the serum from all subjects. We found that four of the 29 subjects with CD had GM-CSF antibodies. BFAHF-2 significantly increased GM-CSF production by PBMCs 


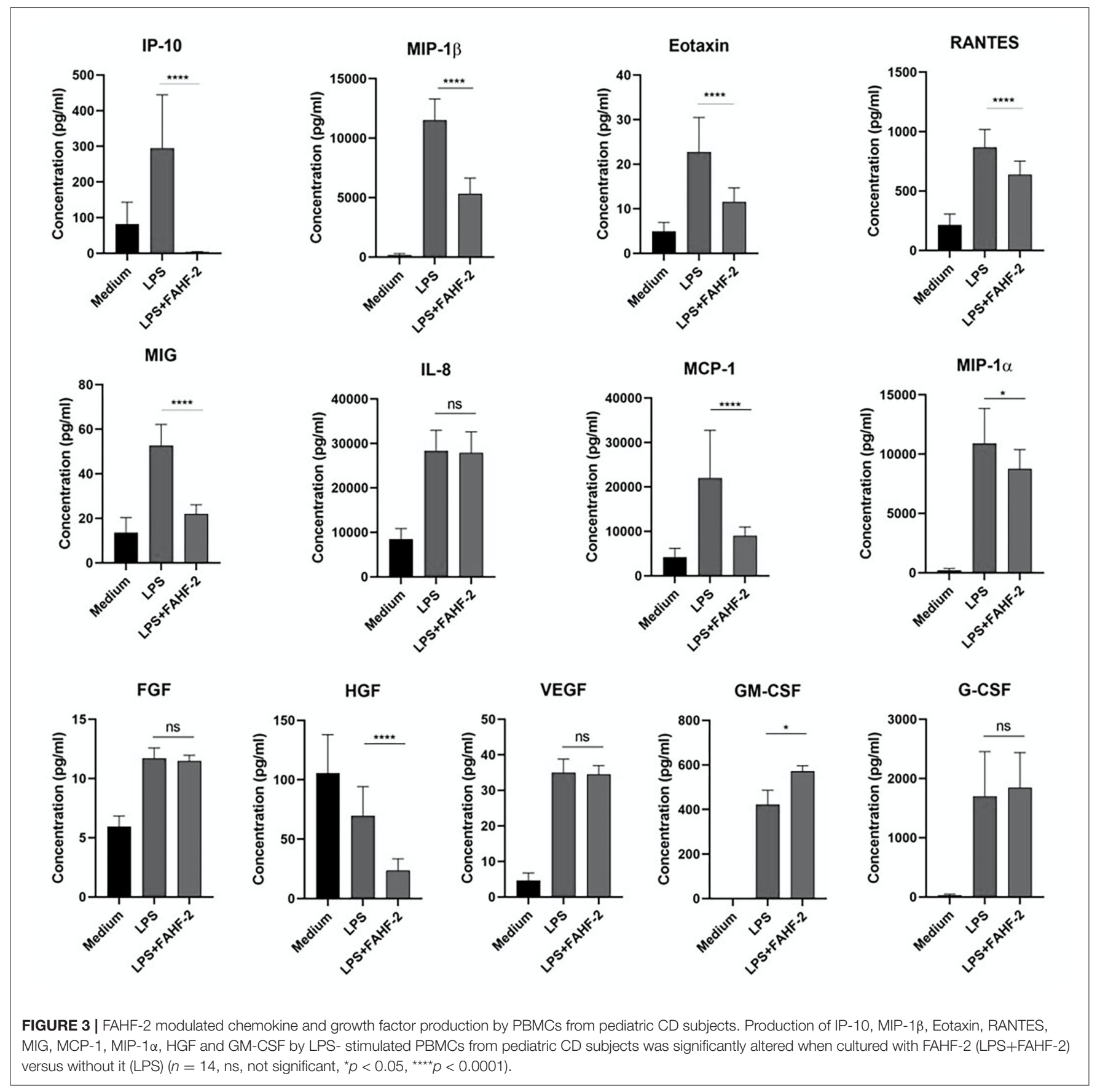

from these 4 subjects $(p<0.05)$ (Figure 5) despite them having antibodies to GM-CSF.

\section{Only B-FAHF-2 and Huang Bai Were Effective at Both Suppressing TNF- $\alpha$ Production and Inducing GM-CSF Production by PBMCs From Pediatric Subjects With CD}

Given the potent suppressive effect of FAHF-2 and B-FAHF2 on TNF- $\alpha$ production, (5) each herb that comprises them
[Prunus mume (Wu Mei), Zanthoxylum bungeanum (Chuan Jiao), Angelica sinensis (Dang Gui), Zingiber officinalis (Gan Jiang), Cinnamomum cassia (Gui Zhi), Phellodendron chinense (Huang Bai), Coptis chinensis (Huang Lian), Panax ginseng (Hong Shen) and Ganoderma lucidum (Ling Zhi)] was tested for the ability to suppress production of TNF- $\alpha$ from LPS stimulated PBMCs from pediatric subjects with CD. LPS caused a marked increase in production of TNF- $\alpha$. B-FAHF-2, Dexamethasone (Dexa), Huang Bai (HB), Huang Lian (HL) and Dang Gui (DG) significantly reduced production of TNF- $\alpha(p<0.0001, p<$ $0.001, p<0.01, p<0.05$, and $p<0.05$ respectively) (Figure 6A). 
A

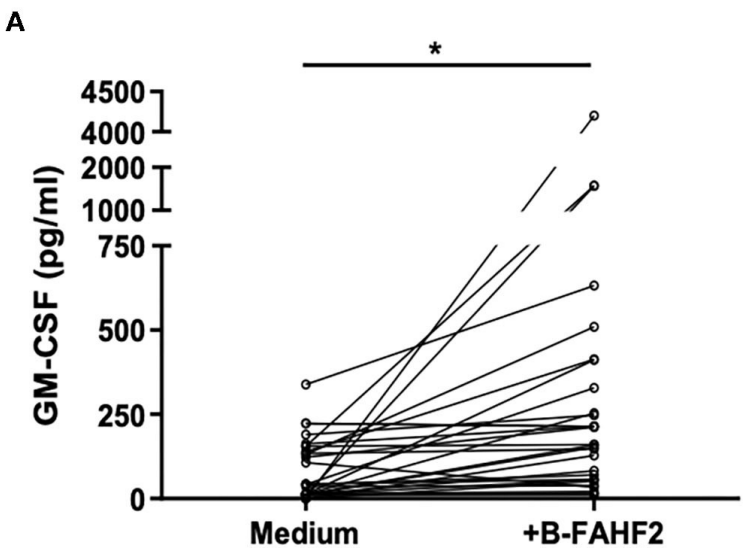

B

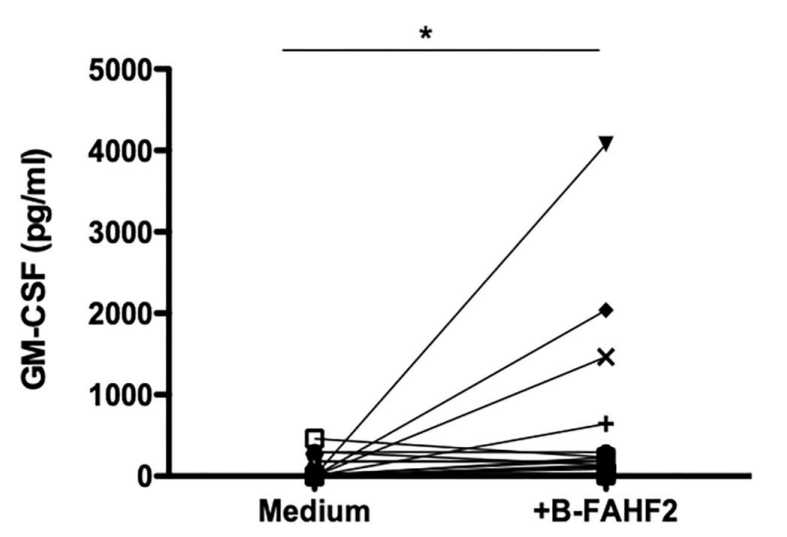

FIGURE 4 | B-FAHF-2 stimulated increased production of GM-CSF by PBMCs and colonic mucosa from pediatric subjects with CD. (A) GM-CSF levels from PBMCs from pediatric subjects with CD cultured in medium or with the addition of B-FAHF-2 $(n=29)$. (B) GM-CSF levels from colonic biopsies from pediatric subjects with CD cultured in medium or with the addition of B-FAHF-2 $(n=20)\left({ }^{*} p<0.05\right)$.

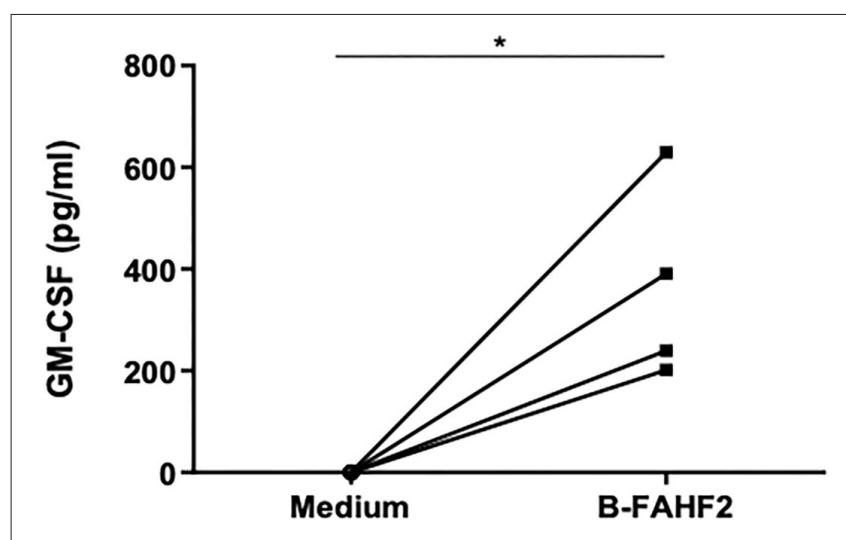

FIGURE 5 | B-FAHF-2 caused increased GM-CSF production by PBMCs from pediatric subjects with $\mathrm{CD}$ who have GM-CSF antibodies. GM-CSF levels from PBMCs from four pediatric subjects with CD and GM-CSF antibodies cultured in medium alone or with the addition of B-FAHF-2 $\left(n=4,{ }^{*} p<0.05\right)$.

We then tested the effect of B-FAHF-2 and each herb that comprises it on the production of GM-CSF. B-FAHF-2, Ling Zhi (LZ) and Huang Bai (HB) induced significantly increased production of GM-CSF by unstimulated PBMCs from pediatric subjects with $\mathrm{CD}(p<0.05, p<0.001$, and $p<0.05$, respectively) (Figure 6B).

Overall, only B-FAHF-2 and Huang Bai (HB) could both decrease TNF- $\alpha$ and increase GM-CSF production by PBMCs unlike any of the other individual herbs.

\section{B-FAHF-2 Alleviated Colitis in a Murine Model}

In the CD45RB hi $\mathrm{T}$ cell transfer model of colitis, treatment with FAHF-2 decreased colitis progression as evidenced by decreased weight loss, histological inflammation and production of TNF$\alpha$, IFN- $\gamma$, IL-6, and IL-17 from the colon (5). Therefore, we assessed the effect of B-FAHF-2 in vivo using the CD45RB hi $T$ cell transfer model of colitis, which exhibits features like those found in $\mathrm{CD}$ including transmural colitis and elevated levels of TNF- $\alpha$. B-FAHF-2 treated mice trended toward less weight loss (Figures 7A,B) and had significantly less colon shortening than untreated controls $(p<0.05)$ (Figure 7C). The B-FAHF2 treated mice had significantly less histological inflammation with less muscularis inflammatory infiltrates, crypt damage, and ulceration $(p<0.05, p<0.05, p=0.0548$, and $p<0.01$, respectively) (Figures $7 \mathbf{D}-\mathbf{F}$ ). The scoring system used takes into account changes in neutrophil infiltrates. Thus, we also looked by IF at $\mathrm{CD} 4^{+} \mathrm{T}$ cell and macrophage infiltrates and found that both were decreased in the B-FAHF-2 treated group (Figure 7F). Inflammatory cytokine production by colonic tissue in B-FAHF-2 treated mice was reduced: TNF- $\alpha$, IFN- $\gamma$, and IL-6 $(p<0.05, p<$ $0.05, p<0.01$, respectively) (Figure 7G). Levels of IL-17 and IL10 were not detectable in both groups (data not shown). Finally, to examine if B-FAHF-2 affects regulatory T cells, we examined TGF- $\beta 1$, TGF- $\beta 2$, Foxp3, and IL- 10 by RT-PCR and found no significant differences between the groups (data not shown).

Thus, B-FAHF-2 alleviates colitis and inhibits the inflammatory milieu associated with colitis.

\section{DISCUSSION}

In this continuation of our studies on FAHF-2 as a potential treatment for $\mathrm{CD}$, we first sought to lessen the pill burden of FAHF-2 required for human trials by purifying the active compounds and then by examining the individual herbs. Our data demonstrates that the purified product, B-FAHF-2, was effective at suppression of TNF- $\alpha$ production by both PBMCs and mucosal biopsies from pediatric subjects with CD at $20 \%$ of the dose of FAHF-2. In a murine model, B-FAHF-2 was effective at alleviating colitis at this same dose. B-FAHF-2 also caused an increase in GM-CSF production even in those subjects with antibodies against it. Of the individual herbs within FAHF-2 and 


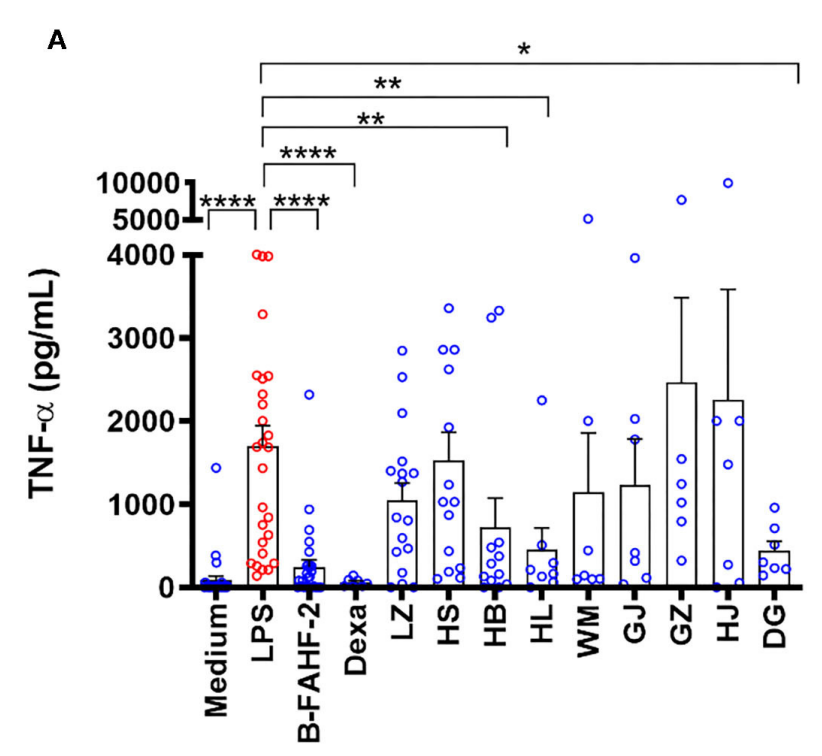

Treatment
B

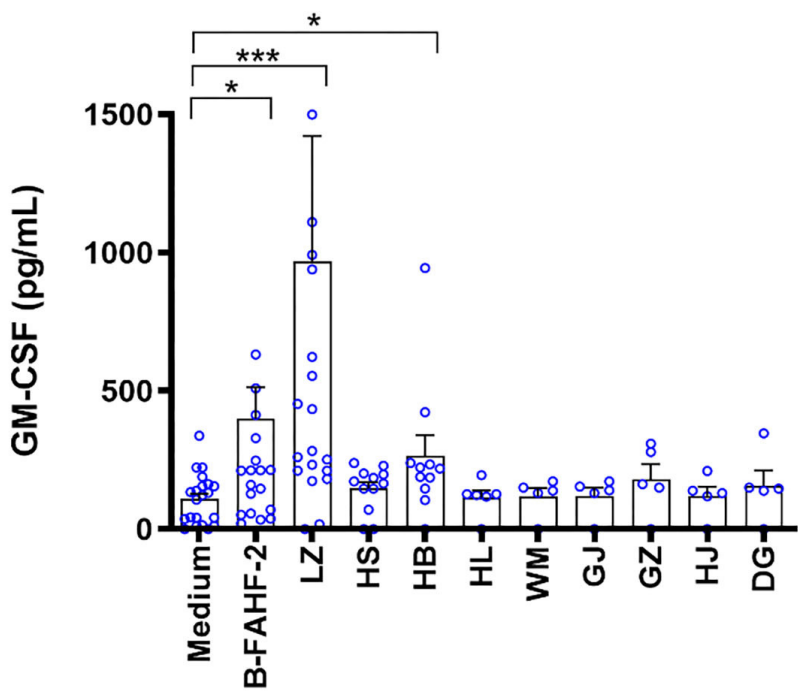

Treatment

FIGURE 6 | Only B-FAHF-2 and Huang Bai suppressed TNF- $\alpha$ and induced GM-CSF production by PBMCs from pediatric subjects with CD. (A) TNF- $\alpha$ levels from PBMCs from pediatric subjects with CD stimulated with LPS and treated with B-FAHF-2, dexamethasone (Dexa), Ganoderma lucidum/Ling Zhi (LZ), Panax ginseng/Hong Shen (HS), Phellodendron chinense/Huang Bai (HB), Coptis chinensis/Huang Lian (HL), Prunus mume/Wu Mei (WM), Zingiber officinalis/Gan Jiang (GJ), Cinnamomum cassia/Gui Zhi (GZ), Zanthoxylum bungeanum/Chuan Jiao (HJ), or Angelica sinensis/Dang Gui (DG). (B) GM-CSF levels from PBMCs from pediatric subjects with CD treated with B-FAHF-2, dexamethasone (Dexa), Ganoderma lucidum/ Ling Zhi (LZ), Panax ginseng/ Hong Shen (HS), Phellodendron chinense/Huang Bai (HB), Coptis chinensis/Huang Lian (HL), Prunus mume/Wu Mei (WM), Zingiber officinalis/Gan Jiang (GJ), Cinnamomum cassia/Gui Zhi (GZ), Zanthoxylum bungeanum/Chuan Jiao (HJ), or Angelica sinensis/Dang Gui (DG) $(n 7-28)\left({ }^{\star \star \star \star} p<0.0001,{ }^{\star \star \star} p<0.001,{ }^{\star \star} p<0.01,{ }^{\star} p<0.05\right.$ as compared with LPS stimulated PBMCs for TNF- $\alpha$ or as compared to medium alone for GM-CSF).

B-FAHF-2, only Huang Bai was effective at both suppressing TNF- $\alpha$ and stimulating GM-CSF production.

Purification of FAHF-2 using butanol extraction did not affect the ability of B-FAHF-2 to suppress TNF- $\alpha$ production ex vivo from human PBMCs and colonic mucosa nor its ability to abrogate colitis in a murine model. The components removed by butanol purification were non-medicinal components including fiber, wax, proteins and starch. Given that B-FAHF-2 has a similar HPLC fingerprint as FAHF-2, and similar effects on TNF- $\alpha$ suppression (5), B-FAHF-2 likely has similar immunomodulatory effects beyond what we have shown here. Importantly, the in-vitro dose of FAHF-2 that inhibited 60$70 \%$ of TNF- $\alpha$ production was $700 \mu \mathrm{g} / \mathrm{mL}$ whereas that of BFAHF-2 is only $60 \mu \mathrm{g} / \mathrm{mL}$. In human trials this equates to a pill burden reduction from 36 pills daily for FAHF-2 to 10 pills maximum per day for B-FAHF-2 (6). This reduction would allow clinical trials to proceed with better participant compliance. An even more purified version of B-FAHF-2 is currently in a phase II trial as an adjunct therapy for children and adults with multiple food allergies (24), and a clinical trial in patients with mild-to-moderate CD, where there is a lack of FDA approved medications, is about to begin (25).

Our results on the effect of FAHF-2 on chemokines and growth factors showed that the formulation is immunomodulatory and not broadly immunosuppressant. Studies have shown that alterations of GM-CSF function may be involved in a subset of IBD cases (14). A recombinant human form of GM-CSF, sargramostim, had positive effects in a pilot study of patients with CD but larger trials did not show improved clinical outcomes $(26,27)$. This is likely due to heterogeneity in pathogenic mechanisms. Therefore, GM-CSF may be an appropriate therapy for a subgroup of CD patients which the larger clinical trial did not target specifically. We demonstrate for the first time, that B-FAHF-2 increases production of GM-CSF from PBMCs and from colonic mucosa from children with CD. The most likely therapeutic target for this increase would be a subset of $\mathrm{CD}$ patients who have been shown to have antibodies against GM-CSF and thus low levels of GM-CSF. In our study population, only 4 children had significant antibodies against GM-CSF and, independent of the presence of antibodies, B-FAHF-2 significantly increased production of GM-CSF by PBMCs from these subjects.

The complexity of Traditional Chinese Medicine makes these therapies particularly difficult to study. Each herbal formulation including B-FAHF-2, incorporates multiple herbs, which in turn are made up of numerous chemicals. The active components of these complex mixtures work synergistically and removal of some components, even if inactive, can destabilize the active components (28). In addition, many of the components may be transformed in vivo into more complex active metabolites that could be eliminated unintentionally. Thus, we were not surprised that only one of the individual herbs, Huang Bai, 


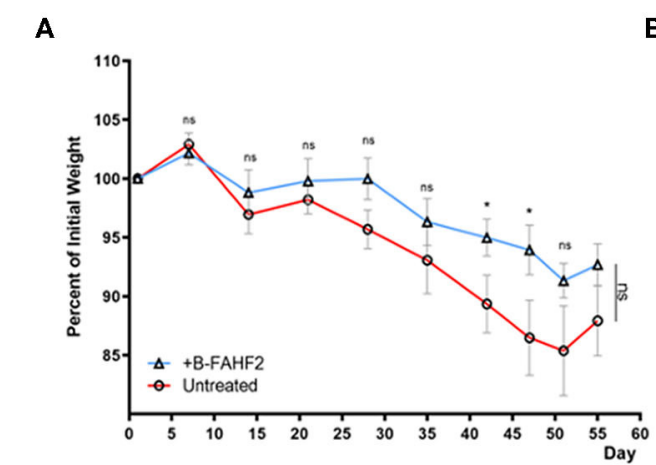

E

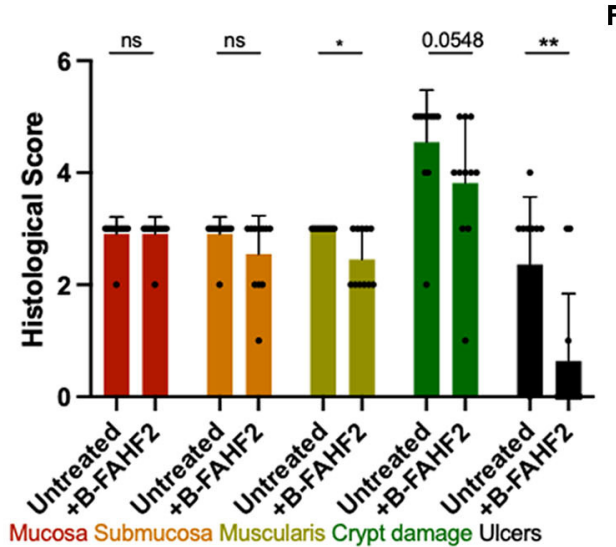

G
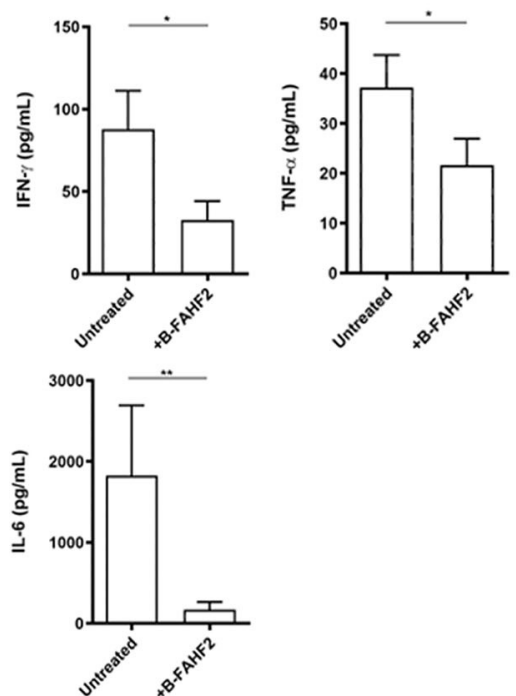

B

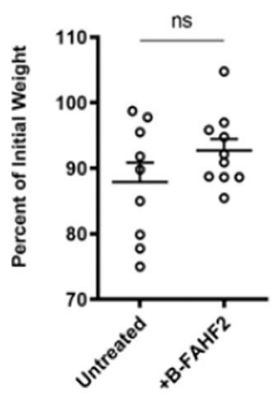

F
Untreated
C

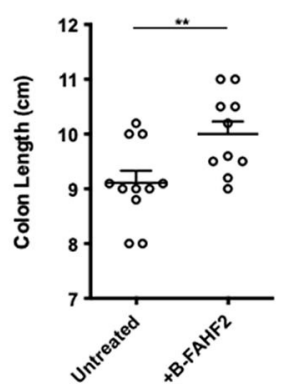

D

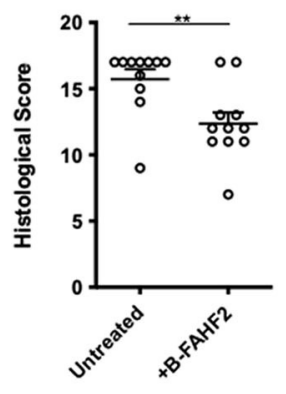

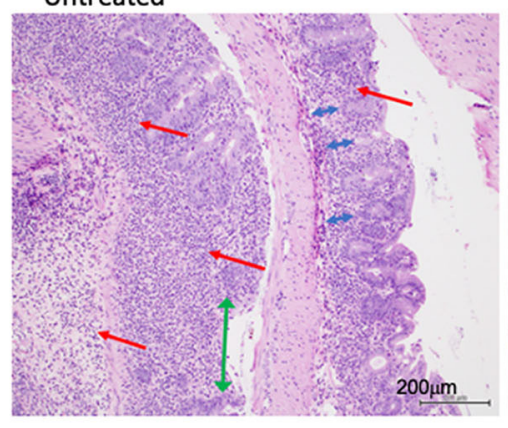

+B-FAHF-2
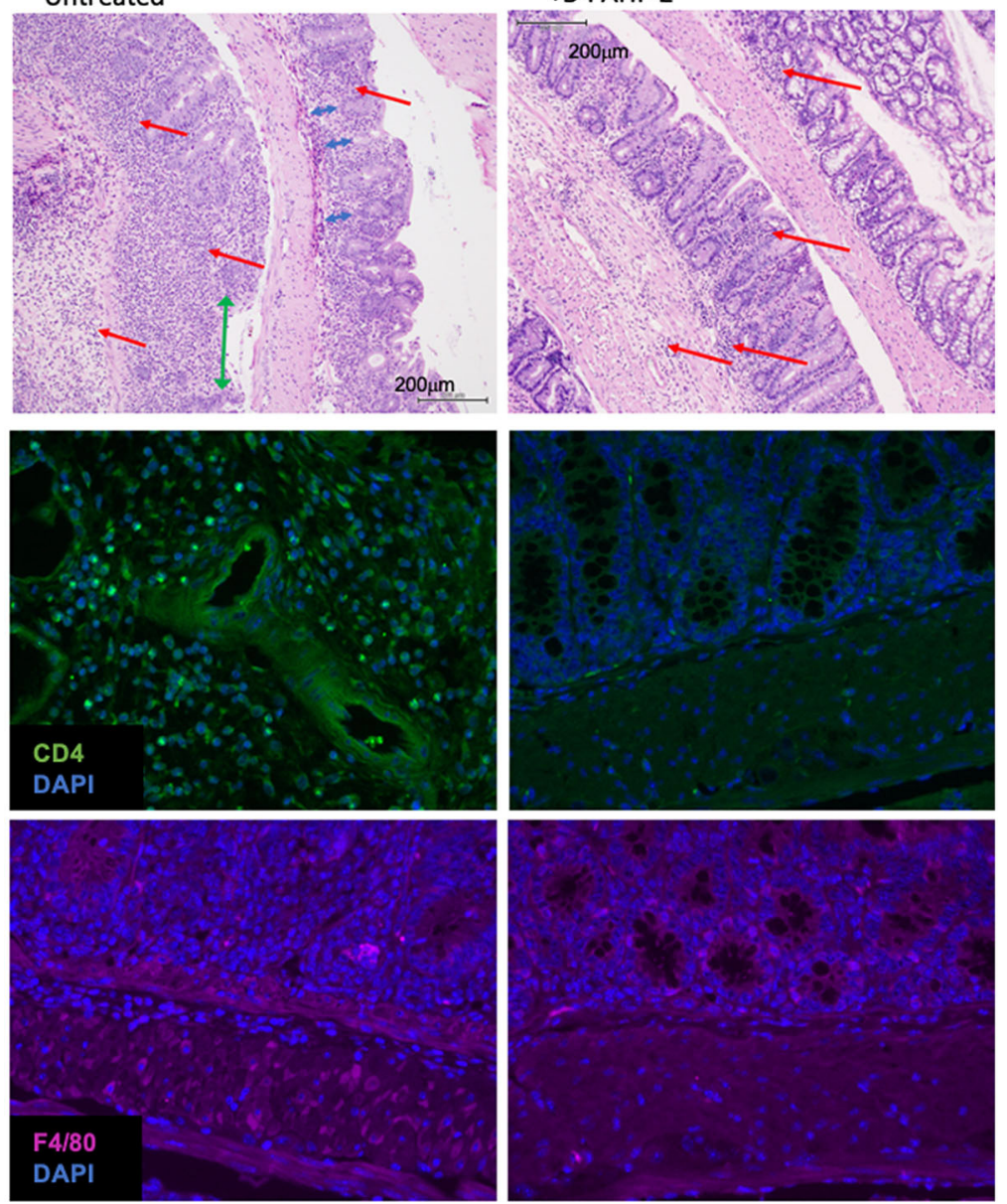

FIGURE 7 | B-FAHF-2 alleviated colitis in the CD45RB hi transfer model. (A) Weight curves of mice treated (+B-FAHF-2) or untreated with B-FAHF-2. (B) The final percentage of initial body weight as measured at the time the mice were sacrificed. (C) Colon length of mice treated (+B-FAHF-2) or untreated with B-FAHF-2. (D) The total histological scores of inflammation of the colon of mice treated (+B-FAHF-2) or untreated. (E) The histological sub-scores for inflammation in the mucosa, submucosa, and muscularis as well as for crypt damage and the extent of ulceration in mice treated (+B-FAHF-2) or untreated. (F) A representative H \& E stained histological figure demonstrating the untreated specimen with marked inflammatory infiltrate including neutrophils (red arrow), loss of crypts (blue arrow), crypt distortion, and ulceration (green arrow) of the mucosa vs. the B-FAHF-2 treated specimen which has significantly less inflammatory infiltrate and disordered crypts but no ulceration and minimal loss of crypts. Immunofluorescence staining for CD4+ T cells and macrophages (F4/80) in untreated and +B-FAHF-2 groups. (G) Cytokine production by colonic mucosa from mice treated (+B-FAHF-2) or untreated with B-FAHF-2 $\left(n=10\right.$ per group, ns, not significant, ${ }^{*} p<0.05$, $\left.{ }^{* *} p<0.01\right)$. 
could both suppress TNF- $\alpha$ and stimulate GM-CSF production although not with the same effect as B-FAHF-2. Other individual herbs were able to either suppress TNF- $\alpha$ or increase GMCSF production but none did both. Interestingly, our group has attempted to isolate active compounds within this complex formulation but has not shown that individual compounds or smaller combinations of them have the same effects as the entire mixture together. In addition to the complexity of the formulation which makes it difficult to study, further challenges to performing clinical trials include obtaining reliable sources for the raw herbal components, ensuring no pesticide or heavy metal contamination, and confirming consistency of the chemical makeup of each batch of herbal medicine. Finally, herbal formulas have often been found to have hepatotoxicity. FAHF2 and B-FAHF-2 were made with the removal of potentially hepatoxic herbs and data in prior murine and human studies have shown no hepatoxicity as evidenced by this formulation receiving an Investigational New Drug status from the $\operatorname{FDA}(8,29)$.

Our study is the next step toward the development of this formulation into an herbal therapy for $\mathrm{CD}$ but additional refinement of the formulation and further larger studies must be performed. This study has several other limitations. We used TNF- $\alpha$ and GM-CSF as surrogate markers for the immunomodulatory capabilities of B-FAHF2 but examination of a multitude of cytokines, chemokines and growth factors should be examined as was done with FAHF-2. In prior studies we examined the mechanism of how FAHF-2 suppressed inflammatory cytokine production and found that the NFкB pathway was inhibited. This is unlikely to explain the mechanism causing increases in GM-CSF production and will need to be investigated in future studies. In addition, we had only a small sample of subjects with antibodies against GM-CSF. The effects of BFAHF-2 in this sub-group should be confirmed in larger patient populations.

\section{CONCLUSIONS}

In conclusion, B-FAHF-2 suppresses production of TNF- $\alpha$, a major inflammatory cytokine involved in the pathogenesis of $\mathrm{CD}$, to a similar extent as FAHF-2 but at a lower dose. BFAHF-2 warrants further clinical investigation to determine its efficacy in CD. B-FAHF-2 also increased production of GM-CSF and thus warrants further investigation in a subgroup of $\mathrm{CD}$ patients who have low levels of or antibodies against GM-CSF.

\section{REFERENCES}

1. Molodecky NA, Soon IS, Rabi DM, Ghali WA, Ferris M, Chernoff G, et al. Increasing incidence and prevalence of the inflammatory bowel diseases with time, based on systematic review. Gastroenterology. (2012) 142:4654.e42. doi: 10.1053/j.gastro.2011.10.001

2. Ouahed J, Spencer E, Kotlarz D, Shouval DS, Kowalik M, Peng K, et al. Very early onset inflammatory bowel disease: a clinical approach with a focus on

\section{DATA AVAILABILITY STATEMENT}

The raw data supporting the conclusions of this article will be made available by the authors, without undue reservation.

\section{ETHICS STATEMENT}

The studies involving human participants were reviewed and approved by Icahn School of Medicine at Mount Sinai Institutional Review Board. Written informed consent to participate in this study was provided by the participants' legal guardian/next of kin. The animal study was reviewed and approved by Icahn School of Medicine at Mount Sinai Institutional Animal Care and Use Committee.

\section{AUTHOR CONTRIBUTIONS}

$\mathrm{XC}$ and JL: validation, formal analysis, writing-original draft, and visualization. YS and SG: methodology, formal analysis, and writing - review and editing. NY: resources, writing-review, and editing. VG, WH, and EC: formal analysis and writingreview and editing. NP, JJ, and KB: specimen collection and writing-review and editing. MD: conceptualization, writingreview and editing. X-ML: conceptualization, writing-review and editing, supervision, project administration, and funding acquisition. DD: conceptualization, formal analysis, resources, writing-review and editing, visualization, supervision, project administration, and funding acquisition. All authors contributed to the article and approved the submitted version.

\section{FUNDING}

This work was partially supported by National Institute of Diabetes Digestive and Kidney Diseases, R03 DK117218 and The Leona M. and Harry B. Helmsley Charitable Trust (to DD), U01 DK1241654 (to SG), and partially supported by NIH 2R01 AT001495-01A1 (to X-ML).

\section{ACKNOWLEDGMENTS}

We appreciate Dr. Elizabeth Spencer for the review of the study statistical analysis. We appreciate the contribution of the Biorepository and Pathology Core at Mount Sinai. This manuscript and data are original and have not been published in other journals. The study was performed according to international, national and institutional rules considering clinical studies and biodiversity rights.

the role of genetics and underlying immune deficiencies. Inflamm Bowel Dis. (2019) 26:820-42. doi: 10.1093/ibd/izz259

3. Ceballos C, Bao R, Dunkin D, Song Y, Li X-M, Benkov K. Complementary and alternative medicine use at a single pediatric inflammatory bowel disease center. Gastroenterol Nurs. (2014) 37:265-71. doi: 10.1097/SGA.0000000000000050

4. China, T.S.P.C.o.T.P.s.R.o. Pharmacopoeia of the People's Republic of China. Version 6. Beijing: People's Medical Publishing House (2005). 
5. Song Y, Dunkin D, Dahan S, Luga A, Ceballos C, Hoffstadter-Thal K, et al. Anti-inflammatory effects of the Chinese herbal formula FAHF2 in experimental and human IBD. Inflamm Bowel Dis. (2014) 20:14453. doi: 10.1097/01.MIB.0000436467.37566.48

6. Srivastava K, Nang N, Chen Y, Lopez-Exposito I, Song Y, Lopez-Exposito I et al. Efficacy, safety and immunological actions of butanol-extracted Food Allergy Herbal Formula-2 on peanut anaphylaxis. Clin Exp Allergy. (2011) 41:582-91. doi: 10.1111/j.1365-2222.2010.03643.x

7. Srivastava KD, Bardina L, Sampson HA, Li X-M. Efficacy and immunological actions of FAHF-2 in a murine model of multiple food allergies. Ann Allergy Asthma Immunol. (2012) 108:351-8.e1. doi: 10.1016/j.anai.201 2.03.008

8. Srivastava KD, Kattan JD, Zou ZM, Li JH, Zhang L, Wallenstein S, et al. The Chinese herbal medicine formula FAHF-2 completely blocks anaphylactic reactions in a murine model of peanut allergy. J Allergy Clin Immunol. (2005) 115:171-8. doi: 10.1016/j.jaci.2004.10.003

9. Srivastava KD, Qu C, Zhang T, Goldfarb J, Sampson HA, Li X-M. Food Allergy Herbal Formula-2 silences peanut-induced anaphylaxis for a prolonged posttreatment period via IFN-gamma-producing CD8+ T cells. J Allergy Clin Immunol. (2009) 123:443-51. doi: 10.1016/j.jaci.2008.12.1107

10. Srivastava KD, Song Y, Yang N, Liu C, Goldberg IE, Nowak-Wegrzyn A, et al. B-FAHF-2 plus oral immunotherapy (OIT) is safer and more effective than OIT alone in a murine model of concurrent peanut/tree nut allergy. Clin Exp Allergy. (2017) 47:1038-49. doi: 10.1111/cea.12936

11. Kattan JD, Srivastava KD, Zou ZM, Goldfarb J, Sampson HA, Li X-M. Pharmacological and immunological effects of individual herbs in the Food Allergy Herbal Formula-2 (FAHF-2) on peanut allergy. Phytother Res. (2008) 22:651-9. doi: 10.1002/ptr.2357

12. Dabritz J, Weinhage T, Varga G, Wirth T, Walscheid K, Brockhausen A, et al. Reprogramming of monocytes by GM-CSF contributes to regulatory immune functions during intestinal inflammation. J Immunol. (2015) 194:242438. doi: 10.4049/jimmunol.1401482

13. Weinhage $T$, Däbritz J, Brockhausen A, Wirth $T$, Brückner $M$, Belz $\mathrm{M}$, et al. Granulocyte macrophage colony-stimulating factor-activated CD39+/CD73+ murine monocytes modulate intestinal inflammation via induction of regulatory T cells. Cell Mol Gastroenterol Hepatol. (2015) 1:43349.e1. doi: 10.1016/j.jcmgh.2015.04.005

14. Han X, Uchida K, Jurickova I, Koch D, Willson T, Samson C, et al. Granulocyte-macrophage colony-stimulating factor autoantibodies in murine ileitis and progressive ileal Crohn's disease. Gastroenterology. (2009) 136:1261-71.e1-3. doi: 10.1053/j.gastro.2008.12.046

15. Korzenik JR, Dieckgraefe BK, Valentine JF, Hausman DF, Gilbert MJ, Sargramostim in Crohn's Disease Study Group. Sargramostim for active Crohn's disease. $N$ Engl J Med. (2005) 352:2193-201. doi: 10.1056/NEJMoa041109

16. Wang J, Jones SM, Pongracic JA, Song Y, Yang N, Sicherer SH, et al. Safety, clinical, and immunologic efficacy of a Chinese herbal medicine (Food Allergy Herbal Formula-2) for food allergy. J Allergy Clin Immunol. (2015) 136:96270.e1. doi: 10.1016/j.jaci.2015.04.029

17. Patil SP, Wang J, Song Y, Noone S, Yang N, Wallenstein S, et al. Clinical safety of Food Allergy Herbal Formula-2 (FAHF-2) and inhibitory effect on basophils from patients with food allergy: extended phase I study. J Allergy Clin Immunol. (2011) 128:1259-602. doi: 10.1016/j.jaci.2011. 06.015

18. Wang JL, Patil SP, Yang N, Ko J, Lee J, Noone S, et al. Safety, tolerability, and immunologic effects of a food allergy herbal formula in food allergic individuals: a randomized, doubleblinded, placebo-controlled, dose escalation, phase 1 study. Ann Allergy Asthma Immunol. (2010) 105:75-7. doi: 10.1016/j.anai.2010. 05.005
19. Xiu S. The Experimental Method of Pharmacology. Beijing: The People's Public Health Publisher (1986).

20. Cooper HS, Murthy SN, Shah RS, Sedergran DJ. Clinicopathologic study of dextran sulfate sodium experimental murine colitis. Lab Invest. (1993) 69:238-49.

21. Ostanin DV, Bao J, Koboziev I, Gray L, Robinson-Jackson SA, KosloskiDavidson $\mathrm{M}$, et al. $\mathrm{T}$ cell transfer model of chronic colitis: concepts, considerations, and tricks of the trade. Am J Physiol Gastrointest Liver Physiol. (2009) 296:G135-46. doi: 10.1152/ajpgi.90462.2008

22. Chen X, Berin MC, Gillespie VL, Sampson HA, David Dunkin. Treatment of intestinal inflammation with epicutaneous immunotherapy requires TGF-beta and IL-10 but not Foxp3(+) Tregs. Front Immunol. (2021) 12:637630. doi: 10.3389/fimmu.2021.637630

23. Zaqout S, Becker LL, Kaindl AM. Immunofluorescence staining of paraffin sections step by step. Front Neuroanat. (2020) 14:582218. doi: $10.3389 /$ fnana.2020.582218

24. E-B-FAHF-2, Multi OIT and Xolair (Omalizumab) for Food Allergy. Bethesda, MD: National Library of Medicine. ClinicalTrials.gov [Internet].

25. Study to Evaluate Safety, Tolerability and Efficacy of Oral E-B-FAHF-2 in Mildto-Moderate Crohn's Disease. Bethesda, MD: National Library of Medicine. ClinicalTrials.gov [Internet].

26. Roth L, Macdonald JK, McDonald JW, Chande N. Sargramostim (GM-CSF) for induction of remission in Crohn's disease. Cochrane Database Syst Rev. (2011) 2011:CD008538. doi: 10.1002/14651858.CD008538.pub2

27. Dieckgraefe BK, Korzenik JR. Treatment of active Crohn's disease with recombinant human granulocyte-macrophage colony-stimulating factor. Lancet. (2002) 360:1478-80. doi: 10.1016/S0140-6736(02)11437-1

28. Liu EH, Qi L-W, Li K, Chu C, Li P. Recent advances in quality control of traditional Chinese medicines. Comb Chem High Throughput Screen. (2010) 13:869-84. doi: 10.2174/138620710793360301

29. Wang J. Treatment of food anaphylaxis with traditional Chinese herbal remedies: from mouse model to human clinical trials. Curr Opin Allergy Clin Immunol. (2013) 13:386-91. doi: 10.1097/ACI.0b013e3283615bc4

Conflict of Interest: X-ML formerly had patents with Herbal Springs for FAHF-2 for treating food allergy. SG is a named inventor of a patent application related to GM-CSF autoantibodies in immune-related colitis. This is filed through the Icahn School of Medicine at Mount Sinai and is currently unlicensed. SG reports consultancy and/or advisory roles for Merck and OncoMed, and research funding from Bristol-Myers Squibb, Celgene, Genentech, Immune Design, Janssen R\&D, Pfizer, Regeneron, and Takeda.

The remaining authors declare that the research was conducted in the absence of any commercial or financial relationships that could be construed as a potential conflict of interest.

Publisher's Note: All claims expressed in this article are solely those of the authors and do not necessarily represent those of their affiliated organizations, or those of the publisher, the editors and the reviewers. Any product that may be evaluated in this article, or claim that may be made by its manufacturer, is not guaranteed or endorsed by the publisher.

Copyright (C) 2021 Chen, Lai, Song, Yang, Gnjatic, Gillespie, Hahn, Chefitz, Pittman, Jossen, Benkov, Dubinsky, Li and Dunkin. This is an open-access article distributed under the terms of the Creative Commons Attribution License (CC BY). The use, distribution or reproduction in other forums is permitted, provided the original author(s) and the copyright owner(s) are credited and that the original publication in this journal is cited, in accordance with accepted academic practice. No use, distribution or reproduction is permitted which does not comply with these terms. 